\title{
Z-mouse : A New Tool in Fuzzy Logic Theory
}

\author{
Fereidoon Shabaninia
}

\author{
School of Electrical and Computer Engineering, Shiraz University, Shiraz, Iran \\ *Corresponding Author: shabaninia@gmail.com
}

Copyright (c) 2014 Horizon Research Publishing All rights reserved.

\begin{abstract}
The concept of a Z-mouse and some of its possible applications are presented in this paper. Z-mouse is considered as a visual means for fuzzy data entry and retrieval. As a graphical tool, Z-mouse has the potential of being used in different applications of fuzzy logic. The focus of this paper is mainly on the introduction of this new tool and some recommended fields in which it may be useful. Cancer images and data dispersion analysis are mentioned as examples.
\end{abstract}

Keywords Z-Mouse, Fuzzy Logic, Graphical Data Entry, Fuzziness, Uncertainty

\section{Introduction}

Since 1965, Fuzzy theory has been widely used in many different applications. Fuzzy logic provides an effective conceptual framework for dealing with the problem of knowledge representation in an environment of uncertainty and imprecision [1]. In realistic problems, there is always some uncertainty associated with the values of the variables or the system parameters [2]. The existing uncertainty highlights the need for a convenient tool which is capable of representing and handling imprecise data. Various approaches for representing uncertainty, have been proposed so far such as: Fuzzy theory, Probability theory, Interval Mathematics, etc. Although the aforementioned approaches have been used in different domains of applicability, the lack of a visual tool which can present the imprecise data in a graphical manner and which is understandable and utilizable by non-specialists, has always existed. Besides, a visual representation of uncertainty can be more effective and more comprehensive rather than a membership function or a probability distribution function. Motivated by the above reasons, the concept of a Z-mouse has been proposed by Professor Lotfi A. Zadeh in 2010 [3]. The purpose of this paper is to introduce Z-mouse as a tool and to recommend some possible applications for it.

The rest of the paper is organized as follows: In section 2, the concept of a Z-mouse and some of its advantages are introduced. Section 3 presents a simple demonstrating example using Z-mouse. In section 4, some suggested applications for Z-mouse are addressed. Finally, the conclusion is given is section 5 .

\section{The Concept of a Z-Mouse}

Typically, computational problems deal with the computation of the value of a variable namely $\mathrm{Y}$, while having access to a collection of input variables namely $\mathrm{X}_{1}, \ldots, \mathrm{X}_{\mathrm{n}}$. and a collection of parameters namely $\mathrm{P}_{1}, \ldots, \mathrm{P}_{\mathrm{m}}$. In realistic problems, there is always some uncertainty about the values of the variables and parameters [2]. The uncertainty may be associated with the measurement error or the imprecision of perception.

Fuzzy Logic has so far proposed two ways of representing uncertainty, fuzziness and fuzzy degrees [2,4]: Words and Membership functions. Firstly, Words have been used as linguistic labels of fuzzy sets such as high, old, warm, etc. Since in linguistics, the exact definitions of the aforementioned words do not exist, words are one of the original sources of uncertainty. This type of uncertainty could specially be seen in the problems where the available information is expressed in the form of words and phrases of a natural language. For example, we may have seen the following information in several cases: 'The temperature is high', 'The pressure of the gas is medium', etc. Hence, the use of the above words will lead us to define the fuzziness associated with the variables. Secondly, Membership functions provide a more mathematical view of fuzziness by defining a membership value for each value the fuzzy variable can take. Mostly used membership functions are trapezoid, triangular, belle shape, etc with a specific predefined mathematical formula for each. A Z-mouse offers a third choice.

Basically, a Z-mouse provides a convenient visual means of fuzzy data entry and retrieval. Simply, a Z-mouse is an electronic implementation of a spray pen [2]. The cursor of the pen is a round fuzzy mark, called an f-mark which will be put on a scale associated with the fuzzy variable or number. A dot identifies the centroid of an f-mark. The cross section of the f-mark is a trapezoidal fuzzy set with adjustable parameters. The colour of the f-mark is also a matter of choice [2]. Fuzziness will be shown graphically by adjusting the size and the colour of the f-mark on the screen of a 
computer. As an example, consider we have a fuzzy variable $X$, which is in the range of $[0,5]$, and we have the information expressed as the phrase " $\mathrm{X}$ is near medium', Figure 1 below, shows the three general ways of representing this fuzziness.

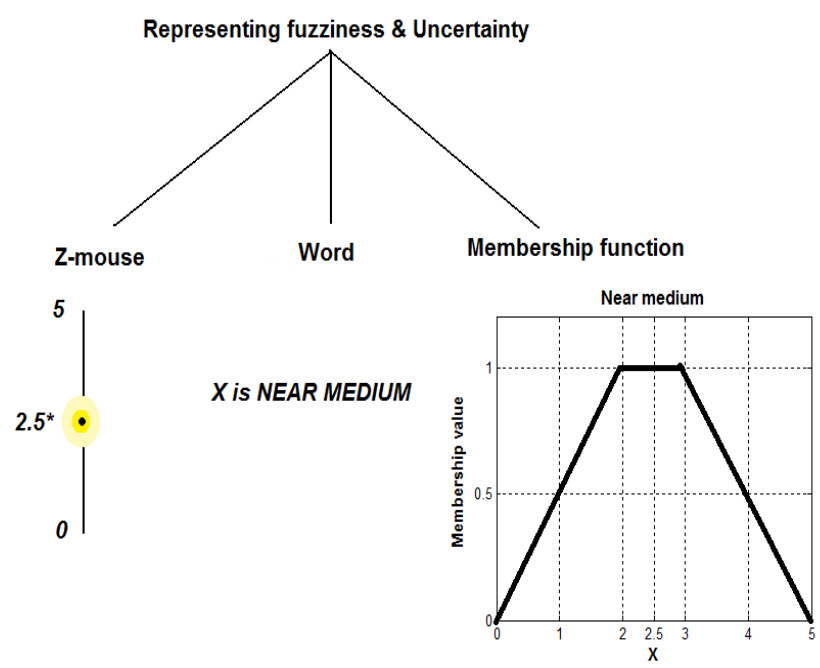

Figure 1. Three ways of representing fuzziness

As a simple illustration, consider the phrase " $\mathrm{X}$ is about 6". The linguistic variable "About" represents the uncertainty and fuzziness of the variable $\mathrm{X}$. Figure 2 shows how $\mathrm{Z}$-mouse performs in a same way as a familiar trapezoidal membership function to present the fuzzy set "About 6".

As it was mentioned, the colour and the size of the f-mark is a matter of choice. Hence, by changing these parameters, one can change the amount of fuzziness and even the shape of the membership function associated with that f-mark. In other words, depending on how dense the centre part is, the shape of the associated membership function may vary. The bigger the black area in the centre of the f-mark is, the longer the upper side of the trapezoid will be. As the black area gets smaller, the length of the upper side of the trapezoid decreases such that in the case that the black area is a tiny dot, the associated membership function would be a triangular instead of a trapezoid. Figure 3 shows how the mapping between Z-mouse and membership function is performed.

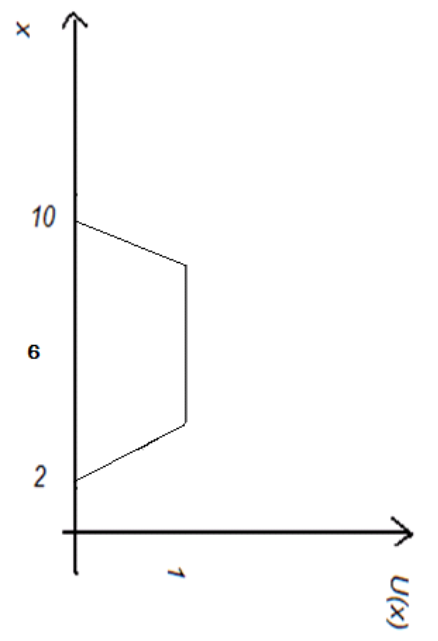

Trapezoidal Membership function

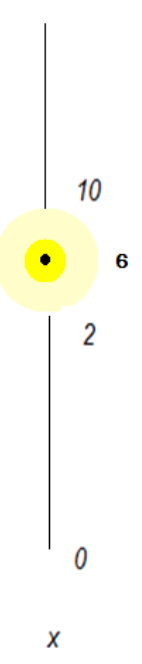

Z-mouse
Figure 2. Representation of " $\mathrm{X}$ is about 6" by MF \& Z-mouse

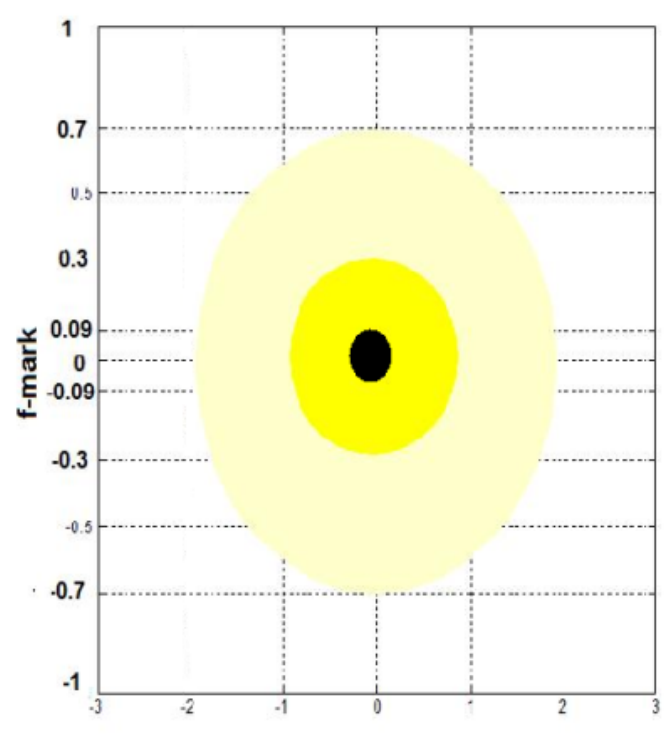

Z-mouse

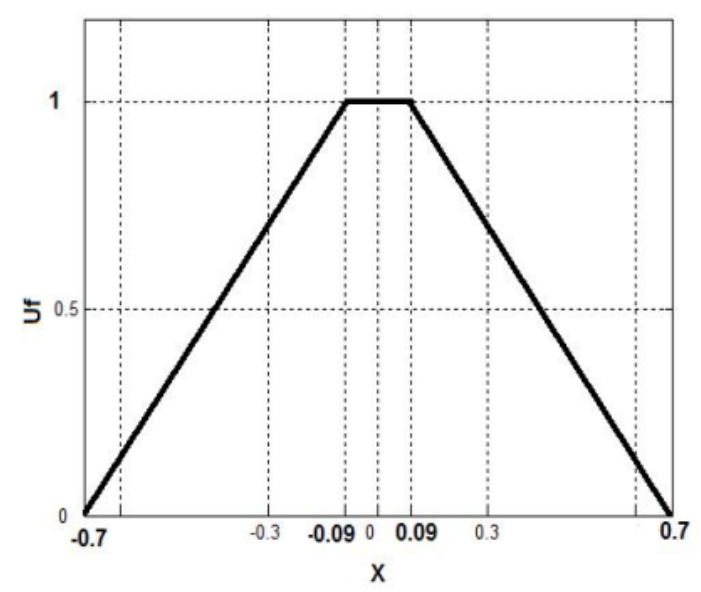

Membership function

Figure 3a. Mapping Z-mouse to MF based on the radius of the central circle: $r=0.09$ 


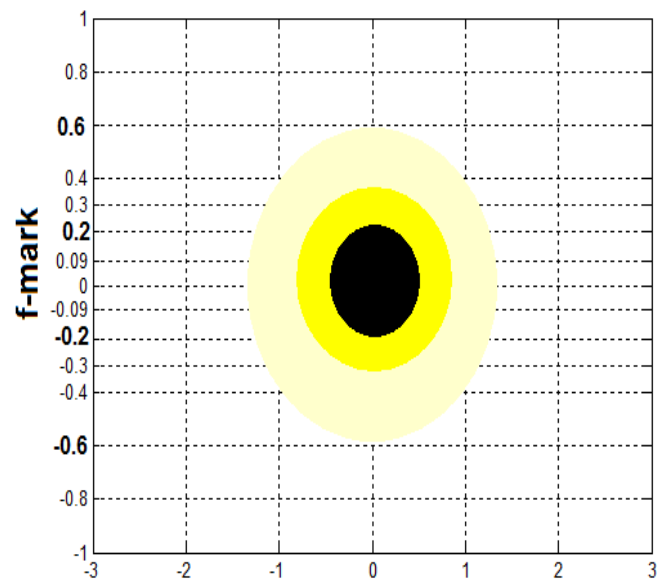

Z-mouse

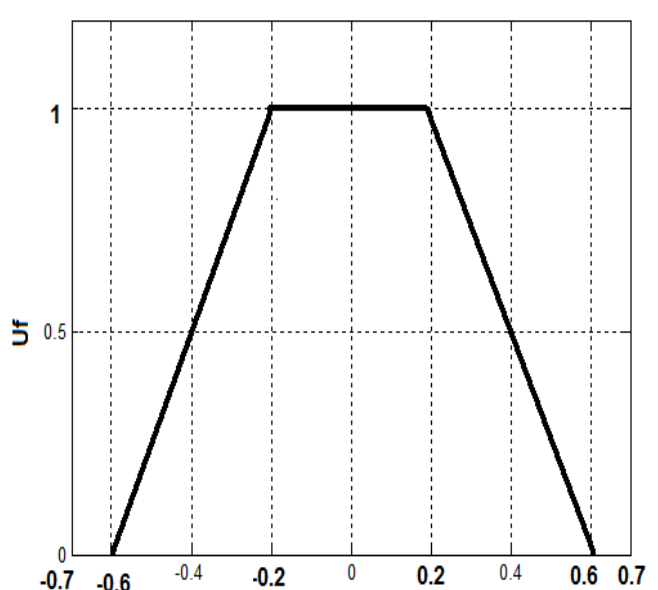

Membership function

Figure 3b. Mapping Z-mouse to MF based on the radius of the central circle: $r=0.2$

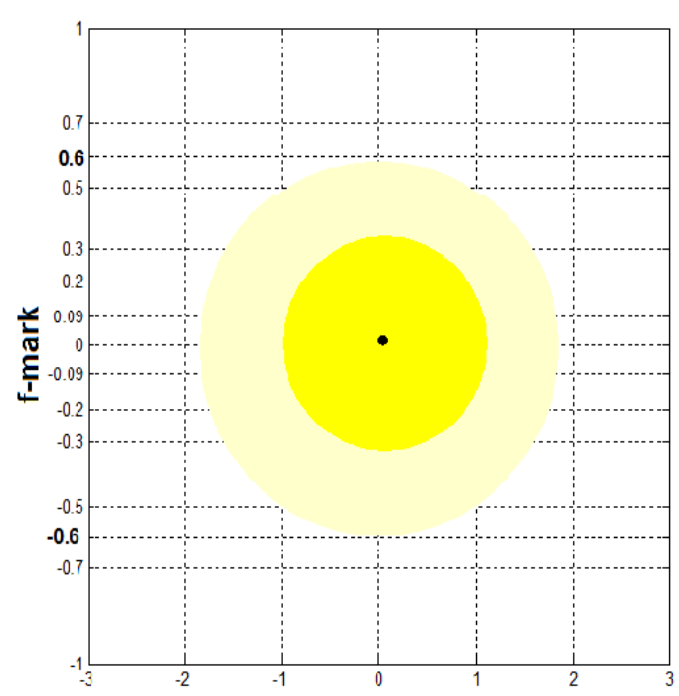

Z-mouse

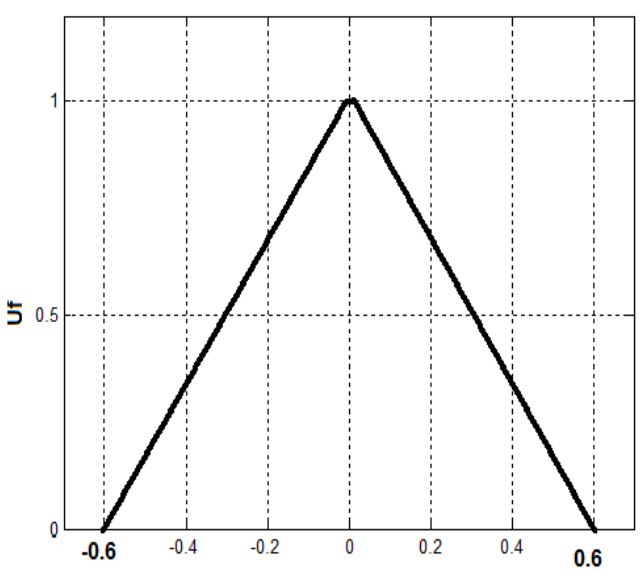

Membership function

Figure 3c. Mapping Z-mouse to MF based on the radius of the central circle: $r<<\xi$

In figures 3.a, 3.b, 3.c, the fuzzy set "near zero" is demonstrated. In Figure 3.a such fuzzy set is defined. Since the radius of the centre black area is 0.09 , the upper side of the trapezoidal membership function (which has the membership value of 1 ) includes the interval [-0.09, 0.09] of the $\mathrm{X}$-axis. In the figure $3 . \mathrm{b}$, the radiuses of the inner and outer circles are different from the first one and hence we have a different trapezoid. In figure 3.c, the definition of the fuzzy set is such that it only accepts a very small neighbourhood of zero as the complete membership. Therefore, the associated membership function much looks like a triangular.

As it was demonstrated, the colours of the f-mark can be chosen to represent uncertainty. To enter the value of a fuzzy variable using a $Z$-mouse, the user marks the scale with an f-mark centring on it. The size of the f-mark is a measure of the user's perception of the fuzziness of the value of the variable. Each fuzzy variable, $X_{\mathrm{i}}$, is associated with a lighted mark on the screen of a computer [2]. The user can also control the position of the mark and move the centroid of the f-mark along the scale. Z-mouse can also be used to represent fuzzy intervals. The following information are presented graphically using Z-mouse in Figure 4 with $150^{*}$ meaning about 150. Consider we have the following information:

1) The height of $A$ is about $150 \mathrm{~cm}$.

2) The height of $B$ is between about $140 \mathrm{~cm}$ and $160 \mathrm{~cm}$. Internally, a Z-mouse translates the f-mark into a membership function. Then, this membership function will be used for the purposes of computation. After the computation is performed, the obtaining result (which may be the value of an output variable) can again be shown graphically as an f-mark on the scale [2].

Some of the advantages of Z-mouse are briefly addressed 
in the following:

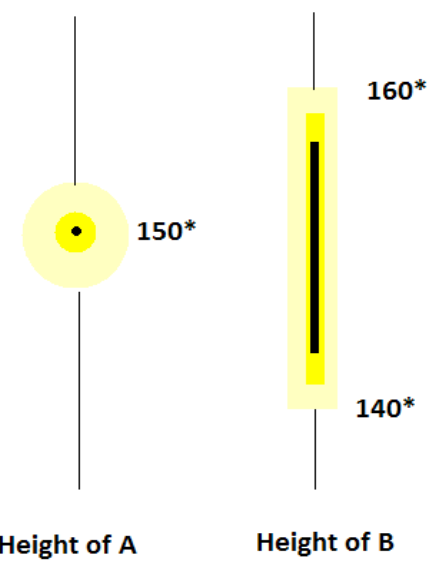

Figure 4. Fuzzy number and Fuzzy interval by Z-mouse

Since Z-mouse serves as a means of visually representing uncertainty or fuzziness of the values of the variables, there is no need for the user to specify the membership function. Therefore, it allows an easy and repeatable way of interacting with non-expert users, since computations are performed internally, unseen by the user [2].

Z-mouse is actually based on a remarkable human capability which is the ability to graduate perceptions without any measurements or computations. For example, usually no one would have difficulties ranking a hotel or a restaurant from 0 to 10 [2]. Hence, in such applications when there is need to allocate a degree to which an object fits a specific class, Z-mouse would be the best means to handle the uncertainty of the one's perception.

Z-mouse can also well handle the case of imprecise probabilities, where there is some fuzziness accompanied with the probability of an event.

\section{Demonstrating Example}

In this section, a simple example is proposed by professor Zadeh in [5], using Z-mouse to show its ease of use in similar applications. Consider we have the following information set:

$\mathrm{P}_{1}$ : Usually, A leaves home for going to work at about 7:00.

$\mathrm{P}_{2}$ : Usually, it takes A near 1 hour to get to work.

The demanded question may be of the following form:

Q: What is the probability that A is at work at 8:15?

We define the fuzzy variables as follows:

$\mathrm{X}_{1}$ : Time of departure

$\mathrm{X}_{2}$ : Travel time

Y: Arrival time

Using the Z-mouse, we will have what is shown in Figure 5. Obviously, this is the visible part by the user. The computations and the mapping of the inserted Z-mouse based fuzzy variables are done internally, unseen by the user and the final result is also shown as an f-mark on the screen.
Computations are done through the use of decision theory and the machinery of CWW [6]. CWW or Computation With Words, is totally explained in [6]. In [4], Professor Zadeh has proposed the method of CWW in which the objects of computation are words, phrases from a natural language. To do so, one needs to first precisate the phrases, define the fuzzy variables and construct some constraints on the variables based on the information given in the phrases. Here are the constructed constraints on the variables $\mathrm{X}_{1}, \mathrm{X}_{2}$ :

1) Probability $\left(X_{1}\right.$ is about 7:00) is usually.

2) Probability $\left(X_{2}\right.$ is near 1:00) is usually.

3) Probability $(\mathrm{Y} \leq 8: 15)$ is?

The computation procedure can be found in [6].

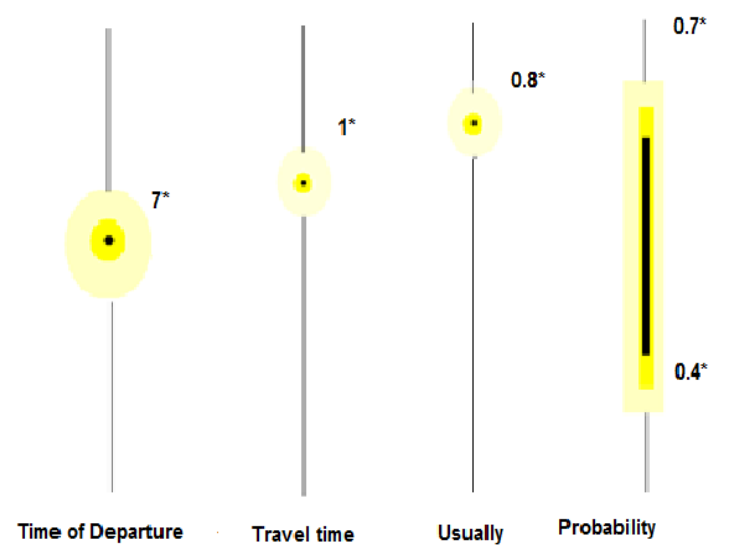

Figure 5. Illustrative Example by Z-mouse

In CWW, one of the most important tasks is to derive the variables and constraints from the phrases. As it can be seen, using Z-mouse to enter the given information in phrases, can be helpful to define the variables and constraints easily. Hence, one of the areas in which Z-mouse can be very effective, is in cases where the phrases and words are carriers of information and there is a vital need to reprocess the information in order to prepare it for the computation with CWW machinery.

\section{Applications}

Besides the examples mentioned, Z-mouse has the potential of being used in a wide range of applications. First, according to the basic concept of Z-mouse, the density of the $\mathrm{f}$-mark is highest in the centre and as we move aside the centre, it becomes sparser. Therefore, Z-mouse uses the dispersion of the data as a measure of fuzziness. This idea could be expanded to other areas. For example, instead of using different colours for different levels of fuzziness and uncertainty, it is possible to use the density of the available data. Figure 6 shows how different data dispersions could have the same role as colours and size in Z-mouse. As it is shown, figure 6 is dense at the centre and sparse at the sides, which means that the data located in the centre are more likely to happen or to belong to a specific class rather than the data located aside. 


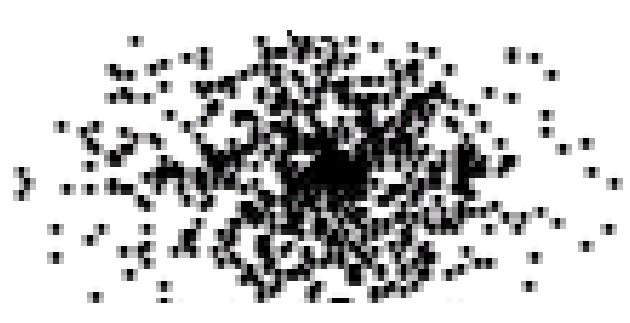

Figure 6. Data dispersion as a measure of fuzziness

Based on what has been mentioned so far, Z-mouse could be used in applications where a series of data are stored and viewed graphically, in order to determine the interval or the area which is in the highest degree of belonging to the associated fuzzy set. To clarify the issue, consider the case of cancer images. In such images, the area which is infected by the cancerous cells, is usually shown by a similar figure as figure 6 . There is an area in the centre which has the highest density and the highest degree of membership to the set "cancerous". Figure 7 shows a sample picture of a tumor.

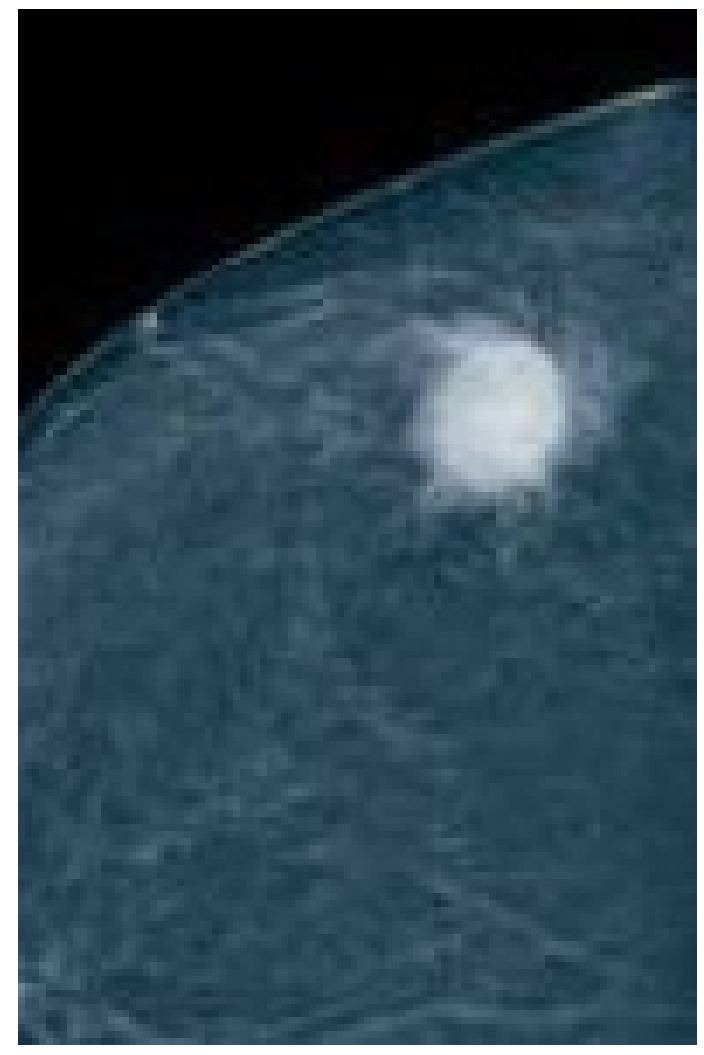

Figure 7. Cancerous cells

As it can be seen from figure 7, the tumor has almost the same properties as an f-mark. Hence, an application area for Z-mouse could be diagnosis and classification of cancerous cells by means of mapping the image into predefined membership functions based on the density of the cells. This procedure can improve the diagnosis and classification of cancer with the help of fuzzy logic.
For a better view, a simple implementation on a cancerous mass has been performed. Figure 8 below, shows a possible cancerous mass.

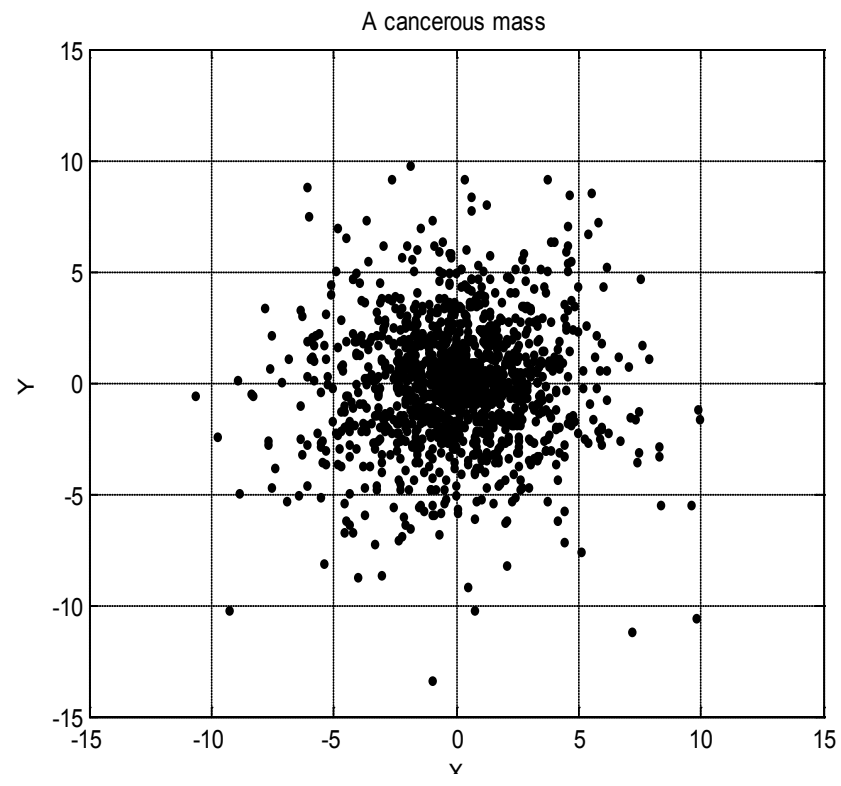

Figure 8. A cancerous area

Based on the dispersion of the data, we have converted the cancerous area into a familiar f-mark on the Z-mouse. Figure 9 shows this procedure.

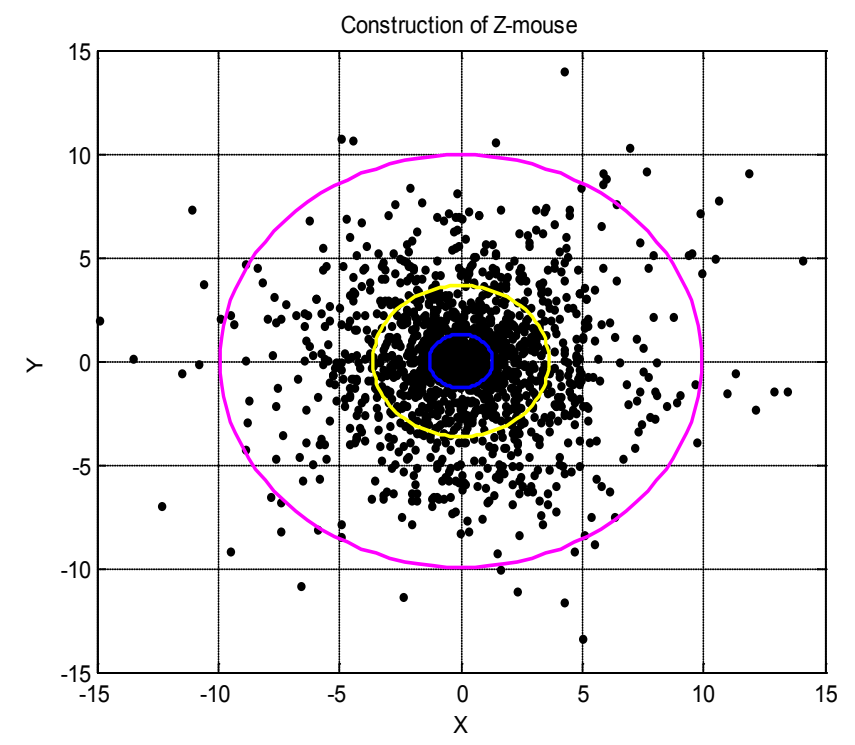

Figure 9. Construction of Z-mouse from the data

In figure 9, the three different colours and sizes of the circles have been constructed based on the density of the data in each section. The obtained result can be viewed as an f-mark. Hence, the only task to do is to map the Z-mouse into a membership function in order to prepare it for further analysis. Based on the radius of each of the circles shown in figure 9, this mapping is performed and demonstrated in figure 10 below. 


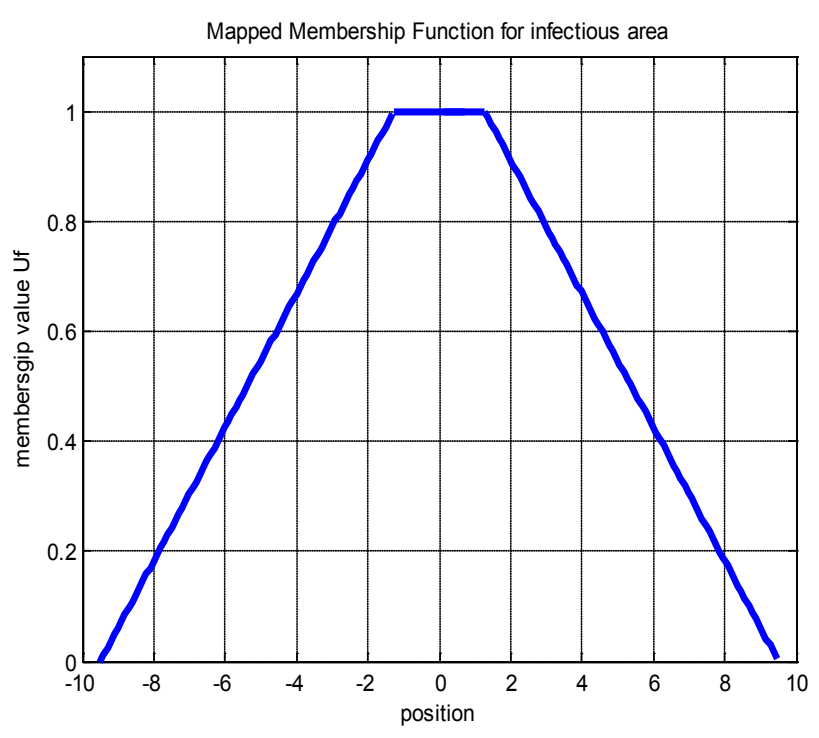

Figure 10. The mapped membership function for infectious area

Since other fuzzy cancer detection techniques such as [7], require high image processing techniques and efforts as well as compiling and programming, it can be concluded that using Z-mouse, as a pre-processor could be of great importance and benefits, since it is easy to implement and the results obtained by $Z$-mouse seem to be very promising. Therefore, it can be beneficial to apply the Z-mouse as means of pre-processing and to get a primary estimation of the infected area, before taking any further steps.

\section{Conclusion}

In this paper, we have presented the concept of a Z-mouse and some of its possible applications. It can be concluded that Z-mouse opens new doors to visually representing uncertain and imprecise data, with the advantage of not getting the user involved with determining the membership functions. Hence, it provides a more convenient environment for the non-experts. It was also shown that Z-mouse can be more than just a tool for data entry, as in the case of cancer images it could be used to diagnose and classify the cancer cells based on the density of the cells in the image.

\section{REFERENCES}

[1] L. A. Zadeh, Fuzzy sets. Information and Control 8,338-353 (1965)

[2] http://mybisc.blogspot.com/2010/08/z-mouse-visual-meansof-entry-and.html

[3] L. A. Zadeh, Foreword to the Special Section on Computing With Words. IEEE Transactions on Fuzzy Systems, VOL. 18, NO. 3, June 2010

[4] http://www.cs.berkeley.edu/ zadeh/presentations.html

[5] Lotfi A. Zadeh, Precisiation of meaning-Toward computation with natural language. International Conference on Information Reuse \& Integration. August 4, 2010

[6] Lotfi A. Zadeh, Toward a generalized theory of uncertainty (GTU) — an outline. Information Sciences 172(2005) 1-40

[7] Devesh D. Nawgaje, Dr. Rajendra D.Kanphade, Implementation of Fuzzy Logic for Detection of Suspicious Masses in Mammograms using DSP TMS320C6711. International Journal of Advanced Engineering \& Application, Jan 2011 Issue 